\title{
Raising and lowering operators of spin-weighted spheroidal harmonics
}

\author{
Abhay G. Shah ${ }^{1}$ - Bernard F. Whiting ${ }^{2,3} \mathbb{D}$
}

Received: 12 January 2016 / Accepted: 16 April 2016 / Published online: 25 May 2016

(C) The Author(s) 2016. This article is published with open access at Springerlink.com

\begin{abstract}
Differential operators for raising and lowering angular momentum for spherical harmonics are used widely in many branches of physics. Less well known are raising and lowering operators for both spin and the azimuthal component of angular momentum (Goldberg et al. in J Math Phys 8:2155, 1967). In this paper we generalize the spin-raising and lowering operators of spin-weighted spherical harmonics to operators linear-in- $\gamma$ for spin-weighted spheroidal harmonics, where $\gamma$ is an additional parameter present in the second order ordinary differential equation governing these harmonics. Constructing these operators has required using all the $\ell-, s$ - and $m$-raising and lowering operators (and various combinations of them) for spin-weighted spherical harmonics, which have been calculated and shown explicitly in this paper. Following a well-defined procedure, the operators given could be generalized to higher powers in $\gamma$.
\end{abstract}

Keywords Spheroidal harmonics · Spin-weighted spheroidal harmonics · Spinweighted spherical harmonics - Spherical harmonics · Raising- and loweringoperators $\cdot$ Angular harmonics

Abhay G. Shah

a.g.shah@soton.ac.uk

Bernard F. Whiting

bernard@phys.ufl.edu

1 School of Mathematics, University of Southampton, Southampton SO17 1BJ, United Kingdom

2 Department of Physics, University of Florida, P.O. Box 118440, Gainesville, Florida 32611-8440, USA

3 GReCO, Institut d'Astrophysique de Paris — UMR 7095 du CNRS, Université Pierre \& Marie Curie, 98 bis boulevard Arago, 75014 Paris, France 


\section{Introduction}

Spin-weighted spherical harmonics, ${ }_{s} Y_{\ell, m}$, occur in many areas of physics-from quantum mechanics to geophysics. By contrast, spin-weighted spheroidal harmonics, ${ }_{s} S_{\ell, m}^{\gamma}$, are much less well known, but they arise naturally in general relativity, for any analysis of the angular dependence of propagating fields on rotating, Kerr black hole space-time backgrounds [2,3], and are most studied in the differential equations governing scalar, linear electromagnetic and gravitational perturbations. When the spin, $s$, of the propagating field is zero, these angular eigenfunctions become the oblate (scalar) spheroidal harmonics [4]. Apart from their application in astrophysics, ordinary spheroidal harmonics $\left(s=0\right.$ of $\left.{ }_{s} S_{\ell, m}^{\gamma}\right), S_{\ell, m}^{\gamma}$, are also used to study molecular physics; for example, they occur in equations describing the hydrogen molecule ion or an electron in a dipole field [5]. Non-zero spin harmonics, the $S_{\ell, m}^{\gamma}$, have been used when studying canonical quantization of electromagnetic field on a Kerr background [6], and Hawking radiation from higher dimensional rotating black holes [7] (also see references in Berti et al. [8] for further applications). Only when the black hole is spherically symmetric, do the full angular eigenfunctions reduce to the spin-weighted spherical harmonics, well known in other areas of Physics.

Though perhaps not very well known, it is actually possible to identify operators for raising and lowering the spin of spin-weighted spheroidal harmonics. In this work we will focus, for the first time, on describing such operators, i.e., for raising and lowering the spin index of the spin-weighted spheroidal harmonics. We explain how these operators can be described exactly, and then will show explicitly how they can be constructed to first order in the parameter perturbing away from the spinweighted spherical harmonics, and lay the groundwork for extending the procedure to higher order. For simplicity, we will generally assume an unwritten factor of $e^{i m \phi}$ throughout, and shall concentrate primarily on the $\theta$-dependence, since the azimuthal eigen-equation is rather trivial.

Spin-weighted spheroidal harmonics satisfy the angular part of Teukolsky's master equation:

$$
\begin{aligned}
{ }_{s} \hat{\square}_{\ell, m}^{\gamma} z= & \frac{1}{\sin \theta} \frac{d}{d \theta}\left(\sin \theta \frac{d z}{d \theta}\right) \\
& -\left(\frac{m^{2}+s^{2}+2 m s \cos \theta}{\sin ^{2} \theta}-\gamma^{2} \cos ^{2} \theta+2 s \gamma \cos \theta-{ }_{s} E_{\ell, m}^{\gamma}\right) z=0
\end{aligned}
$$

where $s$ is the spin weight of the harmonic, and ${ }_{s} E_{\ell, m}^{\gamma}$ represents a discrete set of eigenvalues which allow a solution to be regular in the whole interval $-1 \leq \cos \theta \leq 1$. In the limit $\gamma \rightarrow 0,{ }_{s} E_{\ell, m}^{\gamma}$ is just $\ell(\ell+1)$. As with any second order differential equation, Eq. (1) has two linearly independent solutions, one of which, ${ }_{s} S_{\ell, m}^{\gamma}$ is required to be regular everywhere on the sphere and is used for describing scalar, (masless) neutrino, electromagnetic and gravitational perturbations. In the limit $\gamma \rightarrow 0$, these harmonics are the spin-weighted spherical harmonics, ${ }_{s} Y_{\ell, m}$. In the limit $s \rightarrow 0,{ }_{s} S_{\ell, m}^{\gamma}$ are the 
ordinary spheroidal harmonics, $S_{\ell, m}^{\gamma}$, and ${ }_{s} Y_{\ell, m}$ are the ordinary spherical harmonics, $Y_{\ell, m}$. The ${ }_{s} Y_{\ell, m}$ appear as a solution to the equation

$$
\frac{1}{\sin \theta} \frac{d}{d \theta}\left(\sin \theta \frac{d z}{d \theta}\right)-\left(\frac{m^{2}+s^{2}+2 m s \cos \theta}{\sin ^{2} \theta}-\ell(\ell+1)\right) z=0 .
$$

To build ${ }_{s} Y_{\ell, m}$ for non-zero $s$, one repeatedly applies spin raising and lowering operators on ordinary spherical harmonics, with eigenfunctions of positive and negative values of spin-weight being computed separately:

$$
\begin{aligned}
& { }_{s} Y_{\ell, m}=\partial_{s-1} \partial_{s-2} \ldots \partial_{1} \partial_{0}{ }_{0} Y_{\ell, m},
\end{aligned}
$$

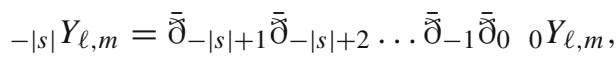

where $s \geq 0$, and for all $s$ we have the definitions:

$$
\begin{aligned}
\partial_{s} & =\frac{-\left(\partial_{\theta}-m \csc \theta-s \cot \theta\right)}{\sqrt{(\ell-s)(\ell+s+1)}}, \\
\bar{\partial}_{s} & =\frac{\left(\partial_{\theta}+m \csc \theta+s \cot \theta\right)}{\sqrt{(\ell+s)(\ell-s+1)}}, \\
{ }_{0} Y_{\ell, m} & =\sqrt{\frac{(2 \ell+1)(\ell-m) !}{4 \pi(\ell+m) !}} P_{\ell}^{m}(\cos \theta) e^{i m \phi} .
\end{aligned}
$$

Here $\partial_{s}$ is the raising operator acting on ${ }_{s} Y_{\ell, m}, \bar{\partial}_{s}$ is the lowering operator acting on ${ }_{s} Y_{\ell, m}$, and $P_{\ell}^{m}$ are the associated Legendre functions. $\partial$ and $\bar{\partial}$ have been given a subscript here to show which spin-weighted quantities they act on. For each $s$, the ${ }_{s} Y_{\ell, m}$ are complete and orthogonal functions on the 2-sphere, and are related to the Wigner D-rotation matrices by

$$
{ }_{s} Y_{\ell, m}(\theta, \phi)=\sqrt{\frac{2 \ell+1}{4 \pi}} D_{-s, m}^{\ell}(\phi, \theta, 0) .
$$

Unlike the way ${ }_{s} Y_{\ell, m}$ are calculated using raising and lowering operators, spinweighted spheroidal harmonics are usually calculated as a sum over ${ }_{s} Y_{\ell, m}$ (see Press and Teukolsky [9]) or as a sum over Jacobi polynomials [10]. In this paper we work on generalizing $\widetilde{\partial}_{s}$ and $\bar{\partial}_{s}$ to operators that raise and lower the spin-weight of spheroidal harmonics, ${ }_{s} S_{\ell, m}^{\gamma}$. That is, if $z={ }_{s} S_{\ell, m}^{\gamma}$ and $y={ }_{s \pm 1} S_{\ell, m}^{\gamma}$ are solutions to two different differential versions of Eq. (1), one being ${ }_{s} \hat{\square}_{\ell, m}^{\gamma} z=0$ and the other being $s_{ \pm 1} \hat{\square}_{\ell, m}^{\gamma} y=0$, then we will find a relation of the form:

$$
y=\alpha z+\beta \partial_{\theta} z
$$

to linear order in $\gamma$, between the solutions ( $y$ and $z$ ) of these equations.

The paper is organized as follows. In Sect. 2, we summarize Whiting's earlier work [11] on finding relations between solutions of two differential equations. In Sect. 3, we 
use this work to calculate the different $\ell$-, $s$ - and $m$-raising and lowering relations of ${ }_{s} Y_{\ell, m}$. In Sect. 4, we build the linear-in- $\gamma s$-raising and lowering operators for ${ }_{s} S_{\ell, m}^{\gamma}$.

\section{Earlier work on relating solutions of two differential equations}

Relations of the general form which we seek have been studied previously by one of us [11] and were extensively used in [12] to show mode stability for the perturbations being discussed here. We now give a brief, and slightly more general, introduction, while more complete details can be found in [11]. Thus, we suppose that $y(x)$ and $z(x)$ satisfy

$$
y^{\prime \prime}+p y^{\prime}+q y=0 \text { and } z^{\prime \prime}+P z^{\prime}+Q z=0,
$$

in which ${ }^{\prime}=d / d x$, and seek conditions that $\alpha$ and $\beta$ must satisfy in order that

$$
y=\alpha z+\beta z^{\prime}
$$

should hold. More specifically, since each of Eq. (7) is second order, two linearly independent solutions exist, say $\left(y_{1}, y_{2}\right)$ and $\left(z_{1}, z_{2}\right)$ respectively, and we will actually demand that the mapping (8) applies more fully, so that:

$$
\begin{aligned}
& y_{1}=\alpha z_{1}+\beta z_{1}^{\prime}, \\
& y_{2}=\alpha z_{2}+\beta z_{2}^{\prime} .
\end{aligned}
$$

That is, every solution for $y$ will map to a solution for $z$. Defining the relevant Wronskians by:

$$
\begin{aligned}
& W\left(y_{1}, y_{2}\right) \equiv W_{y}=y_{1} y_{2}^{\prime}-y_{1}^{\prime} y_{2}=C_{y} e^{-\int p d x} \text { and } \\
& W\left(z_{1}, z_{2}\right) \equiv W_{z}=z_{1} z_{2}^{\prime}-z_{1}^{\prime} z_{2}=C_{z} e^{-\int P d x},
\end{aligned}
$$

where $C_{y}$ and $C_{z}$ are constants, we can invert Eq. (9) to find $\alpha$ and $\beta$ :

$$
\begin{aligned}
& \alpha=\frac{1}{W_{z}}\left(y_{1} z_{2}^{\prime}-y_{2} z_{1}^{\prime}\right), \\
& \beta=-\frac{1}{W_{z}}\left(y_{1} z_{2}-y_{2} z_{1}\right) .
\end{aligned}
$$

Clearly, $\alpha$ and $\beta$ are determined entirely by the solutions they map between. Differentiating (8) once and using Eq. (7) for $z$ we find:

$$
y^{\prime}=\left(\alpha^{\prime}-\beta Q\right) z+\left(\alpha+\beta^{\prime}-\beta P\right) z^{\prime}
$$


Equations (8) and (12) together can be inverted to give $z$ and $z^{\prime}$ in terms of $y$ and $y^{\prime}$. For this we will also need to define:

$$
k=\alpha\left(\alpha+\beta^{\prime}-\beta P\right)-\beta\left(\alpha^{\prime}-\beta Q\right)=\frac{W_{y}}{W_{z}} .
$$

Then

$$
\begin{aligned}
z & =\frac{1}{k}\left(\left(\alpha+\beta^{\prime}-\beta P\right) y-\beta y^{\prime}\right), \\
z^{\prime} & =\frac{1}{k}\left(-\left(\alpha^{\prime}-\beta Q\right) y+\alpha y^{\prime}\right) .
\end{aligned}
$$

Further differentiating Eq. (12), and using both Eq. (7), we can deduce:

$$
\begin{aligned}
0= & \left(\alpha^{\prime \prime}+p \alpha^{\prime}+(q-Q) \alpha-2 Q \beta^{\prime}-Q^{\prime} \beta-(p-P) Q \beta\right) z \\
& +\left(2 \alpha^{\prime}+(p-P) \alpha+\beta^{\prime \prime}+(p-2 P) \beta^{\prime}+(q-Q) \beta-P^{\prime} \beta-(p-P) P \beta\right) z^{\prime},
\end{aligned}
$$

in which each coefficient must separately be zero because of Eq. (9). Thus:

$$
\begin{gathered}
\alpha^{\prime \prime}+p \alpha^{\prime}+(q-Q) \alpha-2 Q \beta^{\prime}-Q^{\prime} \beta-(p-P) Q \beta=0 \\
2 \alpha^{\prime}+(p-P) \alpha+\beta^{\prime \prime}+(p-2 P) \beta^{\prime}+(q-Q) \beta-P^{\prime} \beta-(p-P) P \beta=0 .
\end{gathered}
$$

With the appropriate combination of these, we can now show constructively that:

$$
k^{\prime}=(P-p) k
$$

as already follows from Eqs. (10) and (13) above. In the application we have in mind, $P=p$, so that $k=$ const. We could also check the integrability of Eq. (14) which, with Eq. (17) and some algebra, yields the second of Eqs (16).

Finally we note that the operators in Eq. (7) can be written as:

$$
\begin{gathered}
\partial_{x x}+P \partial_{x}+Q=\left(\partial_{x}-\frac{\alpha}{\beta}+P\right)\left(\partial_{x}+\frac{\alpha}{\beta}\right)+\frac{k}{\beta^{2}} \\
\partial_{x x}+p \partial_{x}+q=\left(\partial_{x}+\frac{\alpha+\beta^{\prime}}{\beta}+p-P\right)\left(\partial_{x}-\frac{\alpha+\beta^{\prime}}{\beta}+P\right)+\frac{k}{\beta^{2}}
\end{gathered}
$$

in which the first order operators are effectively intertwined. 


\section{Spin-weighted spherical harmonics}

Let us denote spin-weighted spherical harmonics of type-1 and type-2 by ${ }_{s} Y_{\ell, m}$ and ${ }_{s} X_{\ell, m}$, being two linearly independent solutions of the following equation,

$$
\frac{1}{\sin \theta} \frac{d}{d \theta}\left(\sin \theta \frac{d z}{d \theta}\right)-\left(\frac{m^{2}+s^{2}+2 m s \cos \theta}{\sin ^{2} \theta}-\ell(\ell+1)\right) z=0
$$

where, in the notation of Sect. 2,

$$
\begin{aligned}
& z_{1}={ }_{s} Y_{\ell, m}, \text { and } \\
& z_{2}={ }_{s} X_{\ell, m} .
\end{aligned}
$$

To build the harmonics of non-zero spin, we begin with spin-weight zero ordinary spherical harmonics (suppressing $e^{i m \phi}$ ):

$$
\begin{aligned}
& { }_{0} Y_{\ell, m}=\sqrt{\frac{(2 \ell+1)(\ell-m) !}{4 \pi(\ell+m) !}} P_{\ell}^{m}(\cos \theta), \\
& { }_{0} X_{\ell, m}=\sqrt{\frac{(2 \ell+1)(\ell-m) !}{4 \pi(\ell+m) !}} Q_{\ell}^{m}(\cos \theta),
\end{aligned}
$$

and apply further $s$-raising and $s$-lowering operators to generate arbitrary spinweighted spherical harmonics:

$$
\begin{aligned}
& \check{\partial}_{s}=\frac{-\left(\partial_{\theta}-m \csc \theta-s \cot \theta\right)}{\sqrt{(\ell-s)(\ell+s+1)}}, \\
& \bar{\partial}_{s}=\frac{\left(\partial_{\theta}+m \csc \theta+s \cot \theta\right)}{\sqrt{(\ell+s)(\ell-s+1)}} .
\end{aligned}
$$

Therefore,

$$
\begin{aligned}
& { }_{s} Y_{\ell, m}=\partial_{s-1} \partial_{s-2} \ldots \partial_{1} \partial_{0}{ }_{0} Y_{\ell, m},
\end{aligned}
$$

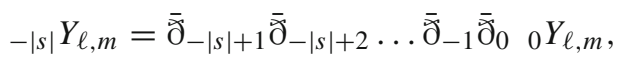

and same holds for ${ }_{s} X_{\ell, m}$.

From Sect. 2 we know that to find relations between solutions of equations

$$
z^{\prime \prime}+P z^{\prime}+Q z=0, \quad y^{\prime \prime}+p y^{\prime}+q y=0
$$


we need to calculate

$$
\begin{aligned}
& \beta=\frac{z_{1} y_{2}-z_{2} y_{1}}{W\left(z_{1}, z_{2}\right)}, \text { and } \\
& \alpha=\frac{-z_{1}^{\prime} y_{2}+z_{2}^{\prime} y_{1}}{W\left(z_{1}, z_{2}\right)}
\end{aligned}
$$

where $W\left(z_{1}, z_{2}\right)$ is defined in (10). One then has

$$
y_{i}=\beta z_{i}^{\prime}+\alpha z_{i}
$$

Finding $\beta$ 's and $\alpha$ 's for various relations, we get

$$
\begin{aligned}
&{ }_{s+1} Y_{\ell, m}= \frac{-1}{\sqrt{(\ell-s)(\ell+s+1)}}\left(\partial_{\theta}-\frac{m}{\sin \theta}-s \cot \theta\right){ }_{s} Y_{\ell, m} \\
&{ }_{s-1} Y_{\ell, m}= \frac{1}{\sqrt{(\ell+s)(\ell-s+1)}}\left(\partial_{\theta}+\frac{m}{\sin \theta}+s \cot \theta\right){ }_{s} Y_{\ell, m} \\
&{ }_{s} Y_{\ell+1, m}= \sqrt{\frac{2 \ell+3}{2 \ell+1}} \frac{(\ell+1) \sin \theta}{\sqrt{\left[(\ell+1)^{2}-m^{2}\right]\left[(\ell+1)^{2}-s^{2}\right]}}\left(\partial_{\theta}+\frac{m s}{(\ell+1) \sin \theta}\right. \\
&+(\ell+1) \cot \theta){ }_{s} Y_{\ell, m} \\
&{ }_{s} Y_{\ell-1, m}= \sqrt{\frac{2 \ell-1}{2 \ell+1} \frac{-\ell \sin \theta}{\sqrt{\left(\ell^{2}-m^{2}\right)\left(\ell^{2}-s^{2}\right)}}\left(\partial_{\theta}-\frac{m s}{\ell \sin \theta}-\ell \cot \theta\right){ }_{s} Y_{\ell, m}} \\
&{ }_{s} Y_{\ell, m+1}=\frac{1}{\sqrt{(\ell-m)(\ell+m+1)}}\left(\partial_{\theta}-\frac{s}{\sin \theta}-m \cot \theta\right){ }_{s} Y_{\ell, m} \\
&{ }_{s} Y_{\ell, m-1}=\frac{-1}{\sqrt{(\ell+m)(\ell-m+1)}}\left(\partial_{\theta}+\frac{s}{\sin \theta}+m \cot \theta\right){ }_{s} Y_{\ell, m}
\end{aligned}
$$

The above six relations are equivalent to Gauss's relations for contiguous functions of the hypergeometric function, ${ }_{2} F_{1}(a, b, c ; z)$. By equating $\partial_{\theta} Y_{\ell, m}$ of two different relations, one can get various numbers of recurrence relations. For example, by equating $\partial_{\theta} Y_{\ell, m}$ in Eqs. (27) and (30), one gets a relations between ${ }_{s} Y_{\ell, m},{ }_{s+1} Y_{\ell, m}$ and ${ }_{s} Y_{\ell-1, m}$. By repeated application of this procedure, various relations can be formed between different ${ }_{s+i} Y_{\ell+j, m+k}$ (where $i, j, k$ are integers). Finally, as an example of 
Eq. (18) we show:

$$
\begin{aligned}
& \partial_{\theta \theta}+\cot \theta \partial_{\theta}-\frac{m^{2}+s^{2}+2 m s \cos \theta}{\sin ^{2} \theta}+\ell(\ell+1) \\
&=\left(\partial_{\theta}+\frac{m}{\sin \theta}+(s+1) \cot \theta\right)\left(\partial_{\theta}-\frac{m}{\sin \theta}-s \cot \theta\right)+(\ell-s)(\ell+s+1) \\
&=\left(\partial_{\theta}-\frac{m}{\sin \theta}-(s-1) \cot \theta\right)\left(\partial_{\theta}+\frac{m}{\sin \theta}+s \cot \theta\right)+(\ell+s)(\ell-s+1) \\
&=\left(\partial_{\theta}-\frac{m s}{(\ell+1) \sin \theta}-\ell \cot \theta\right)\left(\partial_{\theta}+\frac{m s}{(\ell+1) \sin \theta}+(\ell+1) \cot \theta\right) \\
&+\frac{\left((\ell+1)^{2}-m^{2}\right)\left((\ell+1)^{2}-s^{2}\right)}{(\ell+1)^{2} \sin { }^{2} \theta} \\
&=\left(\partial_{\theta}+\frac{m s}{\ell \sin \theta}+(\ell+1) \cot \theta\right)\left(\partial_{\theta}-\frac{m s}{\ell \sin \theta}-\ell \cot \theta\right)+\frac{\left(\ell^{2}-m^{2}\right)\left(\ell^{2}-s^{2}\right)}{\ell^{2} \sin ^{2} \theta} \\
&=\left(\partial_{\theta}+\frac{s}{\sin \theta}+(m+1) \cot \theta\right)\left(\partial_{\theta}-\frac{s}{\sin \theta}-m \cot \theta\right)+(\ell-m)(\ell+m+1) \\
&=\left(\partial_{\theta}-\frac{s}{\sin \theta}-(m-1) \cot \theta\right)\left(\partial_{\theta}+\frac{s}{\sin \theta}+m \cot \theta\right)+(\ell+m)(\ell-m+1)
\end{aligned}
$$

\section{Spin-weighted spheroidal harmonics}

Let us denote the spin-weighted spheroidal harmonics of type-1 and type-2 by ${ }_{s} S_{\ell, m}^{\gamma}$ and ${ }_{s} T_{\ell, m}^{\gamma}$, being two linearly independent solutions of the following equation,

$$
\begin{aligned}
& \frac{1}{\sin \theta} \frac{d}{d \theta}\left(\sin \theta \frac{d z}{d \theta}\right) \\
& -\left(\frac{m^{2}+s^{2}+2 m s \cos \theta}{\sin ^{2} \theta}-\gamma^{2} \cos ^{2} \theta+2 s \gamma \cos \theta-{ }_{s} E_{\ell, m}^{\gamma}\right) z=0 .
\end{aligned}
$$

To linear-in- $\gamma$, expressions for ${ }_{s} S_{\ell, m}^{\gamma}$ and ${ }_{s} E_{\ell, m}^{\gamma}$ have been given by Press and Teukolsky [9]. We write:

$$
\begin{aligned}
& { }_{s} S_{\ell, m}^{\gamma}={ }_{s} Y_{\ell, m}+\gamma_{s} b_{\ell, \ell+1}^{m}{ }_{1} Y_{\ell+1, m}+\gamma_{s} b_{\ell, \ell-1}^{m}{ }_{\ell} Y_{\ell-1, m}+O\left(\gamma^{2}\right), \text { and } \\
& { }_{s} T_{\ell, m}^{\gamma}={ }_{s} X_{\ell, m}+\gamma_{s} b_{\ell, \ell+1}^{m} X_{\ell+1, m}+\gamma_{s} b_{\ell, \ell-1}^{m} X_{\ell-1, m}+O\left(\gamma^{2}\right) .
\end{aligned}
$$


We first look at the Wronskian to $O(\gamma)$,

$$
\begin{aligned}
W\left({ }_{s} S_{\ell, m}^{\gamma},{ }_{s} T_{\ell, m}^{\gamma}\right)= & \left({ }_{s} Y_{\ell, m s} X_{\ell, m}^{\prime}-{ }_{s} X_{\ell, m s} Y_{\ell, m}^{\prime}\right) \\
& +\gamma{ }_{s} b_{\ell, \ell+1}^{m}\left(-{ }_{s} Y_{\ell+1, m}^{\prime} X_{\ell, m}+{ }_{s} X_{\ell+1, m}^{\prime} Y_{\ell, m}\right) \\
& +\gamma{ }_{s} b_{\ell, \ell+1}^{m}\left(-{ }_{s} Y_{\ell, m}^{\prime} X_{\ell+1, m}+{ }_{s} X_{\ell, m}^{\prime} s Y_{\ell+1, m}\right) \\
& +\gamma{ }_{s} b_{\ell, \ell-1}^{m}\left(-{ }_{s} Y_{\ell-1, m}^{\prime} X_{\ell, m}+{ }_{s} X_{\ell-1, m}^{\prime} Y_{\ell, m}\right) \\
& +\gamma{ }_{s} b_{\ell, \ell-1}^{m}\left(-{ }_{s} Y_{\ell, m}^{\prime} X_{\ell-1, m}+{ }_{s} X_{\ell, m}^{\prime}{ }_{\ell} Y_{\ell-1, m}\right)+O\left(\gamma^{2}\right) .
\end{aligned}
$$

In the above equation, the expression in the first parenthesis is a Wronskian which we denote by ${ }_{s} w_{\ell, m}$, the expression in the second parenthesis is $\alpha_{s} w_{\ell+1, m}$ of $\left({ }_{s} Y_{\ell+1, m} \rightarrow\right.$ $\left.{ }_{s} Y_{\ell, m}\right)$, the expression in the third parenthesis is $\alpha_{s} w_{\ell, m}$ of $\left({ }_{s} Y_{\ell, m} \rightarrow{ }_{s} Y_{\ell+1, m}\right)$, the expression in the fourth parenthesis is $\alpha_{s} w_{\ell-1, m}$ of $\left({ }_{s} Y_{\ell-1, m} \rightarrow{ }_{s} Y_{\ell, m}\right)$, and the expression in the fifth parenthesis is $\alpha_{s} w_{\ell, m}$ of $\left({ }_{s} Y_{\ell, m} \rightarrow{ }_{s} Y_{\ell-1, m}\right)$, all of which are known. The analytical expression of $b$ 's and $\alpha$ 's are

$$
\begin{gathered}
{ }_{s} b_{\ell, \ell+1}^{m}=-\frac{s \sqrt{\left[(\ell+1)^{2}-m^{2}\right]\left[(\ell+1)^{2}-s^{2}\right]}}{(\ell+1)^{2} \sqrt{(2 \ell+1)(2 \ell+3)}} \\
{ }_{s} b_{\ell, \ell-1}^{m}=\frac{s \sqrt{\left(\ell^{2}-m^{2}\right)\left(\ell^{2}-s^{2}\right)}}{\ell^{2} \sqrt{(2 \ell-1)(2 \ell+1)}} \\
{ }_{s} w_{\ell, m}=W\left({ }_{s} Y_{\ell, m},{ }_{s} X_{\ell, m}\right)=\frac{-(2 \ell+1) \csc \theta}{4 \pi} \\
\alpha_{(s, \ell+1, m) \rightarrow(s, \ell, m)=} \sqrt{\frac{2 \ell+1}{2 \ell+3}} \frac{(\ell+1) \sin \theta}{\sqrt{\left[(\ell+1)^{2}-m^{2}\right]\left[(\ell+1)^{2}-s^{2}\right]}}\left(\frac{m s}{(\ell+1) \sin \theta}\right. \\
\alpha_{(s, \ell, m) \rightarrow(s, \ell+1, m)=} \sqrt{\frac{2 \ell+3}{2 \ell+1}} \frac{(\ell) \cot \theta),}{\sqrt{\left[(\ell+1)^{2}-m^{2}\right]\left[(\ell+1)^{2}-s^{2}\right]}}\left(\frac{m s}{(\ell+1) \sin \theta}\right. \\
+(\ell+1) \cot \theta), \\
\alpha_{(s, \ell-1, m) \rightarrow(s, \ell, m)}=\sqrt{\frac{2 \ell+1}{2 \ell-1}} \frac{\ell \sin \theta}{\sqrt{\left(\ell^{2}-m^{2}\right)\left(\ell^{2}-s^{2}\right)}}\left(\frac{m s}{\ell \sin \theta}+\ell \cot \theta\right) \\
\sqrt{\frac{2 \ell-1}{2 \ell+1}} \frac{\ell \sin \theta}{\sqrt{\left(\ell^{2}-m^{2}\right)\left(\ell^{2}-s^{2}\right)}\left(\frac{m s}{\ell \sin \theta}+\ell \cot \theta\right)} \\
\alpha_{(s, \ell, m) \rightarrow(s, \ell-1, m)}
\end{gathered}
$$

Using these together, we get

$$
W\left({ }_{s} S_{\ell, m}^{\gamma},{ }_{s} T_{\ell, m}^{\gamma}\right)=-\frac{2 \ell+1}{4 \pi \sin \theta}\left(1+\frac{2 m s^{2} \gamma}{\ell^{2}(\ell+1)^{2}}\right)+O\left(\gamma^{2}\right) .
$$


We now wish to find the s-raising operator of ${ }_{s} S_{\ell, m}^{\gamma}$ to linear-order-in- $\gamma$. We start with finding the $\beta$ and $\alpha$ in the following relation

$$
y=\beta z^{\prime}+\alpha z+O\left(\gamma^{2}\right)
$$

where $y={ }_{s+1} S_{\ell, m}^{\gamma},{ }_{s+1} T_{\ell, m}^{\gamma}$, and $z={ }_{s} S_{\ell, m}^{\gamma},{ }_{s} T_{\ell, m}^{\gamma}$.

We first look at $\beta$ :

$$
\begin{aligned}
& \beta=\frac{{ }_{s} S_{\ell, m}^{\gamma} s+1 T_{\ell, m}^{\gamma}-{ }_{s} T_{\ell, m}^{\gamma} s+1}{{ }^{\gamma} S_{\ell, m}^{\gamma}} \\
& =-\frac{4 \pi \sin \theta}{2 \ell+1}\left(1-\frac{2 m s^{2} \gamma}{\ell^{2}(\ell+1)^{2}}\right)\left({ }_{s} S_{\ell, m}^{\gamma} s+1 T_{\ell, m}^{\gamma}-{ }_{s} T_{\ell, m}^{\gamma} s+1 S_{\ell, m}^{\gamma}\right)+O\left(\gamma^{2}\right) \text {. }
\end{aligned}
$$

To calculate the above, we need

$$
\begin{aligned}
& \left({ }_{s} S_{\ell, m}^{\gamma} s+1 T_{\ell, m}^{\gamma}-{ }_{s} T_{\ell, m}^{\gamma} s+1 S_{\ell, m}^{\gamma}\right) \\
& =\left({ }_{s} Y_{\ell, m}{ }_{s+1} X_{\ell, m}-{ }_{s} X_{\ell, m s+1} Y_{\ell, m}\right) \\
& +\gamma_{s} b_{\ell, \ell+1}^{m}\left({ }_{s} Y_{\ell+1, m} s+1 X_{\ell, m}-{ }_{s} X_{\ell+1, m} s+1 Y_{\ell, m}\right) \\
& +\gamma_{s} b_{\ell, \ell-1}^{m}\left({ }_{s} Y_{\ell-1, m}{ }_{s+1} X_{\ell, m}-{ }_{s} X_{\ell-1, m}{ }_{s+1} Y_{\ell, m}\right) \\
& +\gamma_{s+1} b_{\ell, \ell+1}^{m}\left({ }_{s} Y_{\ell, m} s+1 X_{\ell+1, m}-{ }_{s} X_{\ell, m} s+1 Y_{\ell+1, m}\right) \\
& +\gamma_{s+1} b_{\ell, \ell-1}^{m}\left({ }_{s} Y_{\ell, m s+1} X_{\ell-1, m}-{ }_{s} X_{\ell, m} s+1 Y_{\ell-1, m}\right) \text {. }
\end{aligned}
$$

We next look at $\alpha$ :

$$
\begin{aligned}
& \alpha=\frac{-{ }_{s} S_{\ell, m}^{\gamma^{\prime} s+1} T_{\ell, m}^{\gamma}+{ }_{s} T_{\ell, m}^{\gamma \prime} s+1}{{ }^{\prime} S_{\ell, m}^{\gamma}} \\
& =-\frac{4 \pi \sin \theta}{2 \ell+1}\left(1-\frac{2 m s^{2} \gamma}{\ell^{2}(\ell+1)^{2}}\right)\left(-{ }_{s} S_{\ell, m}^{\gamma^{\prime} s+1} T_{\ell, m}^{\gamma}+{ }_{s} T_{\ell, m}^{\gamma \prime} s+1 S_{\ell, m}^{\gamma}\right)+O\left(\gamma^{2}\right) \text {. }
\end{aligned}
$$

To calculate the above, we need

$$
\begin{aligned}
& \left(-{ }_{s} S_{\ell, m}^{\gamma^{\prime} s+1} T_{\ell, m}^{\gamma}+{ }_{s} T_{\ell, m}^{\gamma^{\prime} s+1} S_{\ell, m}^{\gamma}\right) \\
& =\left(-{ }_{s} Y_{\ell, m}^{\prime} s+1 X_{\ell, m}+{ }_{s} X_{\ell, m}^{\prime} s+1 Y_{\ell, m}\right) \\
& +\gamma{ }_{s} b_{\ell, \ell+1}^{m}\left(-{ }_{s} Y_{\ell+1, m}^{\prime} s+1 X_{\ell, m}+{ }_{s} X_{\ell+1, m}^{\prime} s+1 Y_{\ell, m}\right) \\
& +\gamma{ }_{s} b_{\ell, \ell-1}^{m}\left(-{ }_{s} Y_{\ell-1, m}^{\prime} s+1 X_{\ell, m}+{ }_{s} X_{\ell-1, m}^{\prime} s+1 Y_{\ell, m}\right) \\
& +\gamma_{s+1} b_{\ell, \ell+1}^{m}\left(-{ }_{s} Y_{\ell, m}^{\prime} s+1 X_{\ell+1, m}+{ }_{s} X_{\ell, m}^{\prime} s+1 Y_{\ell+1, m}\right) \\
& +\gamma_{s+1} b_{\ell, \ell-1}^{m}\left(-{ }_{s} Y_{\ell, m}^{\prime} s+1 X_{\ell-1, m}+{ }_{s} X_{\ell, m}^{\prime} s+1 Y_{\ell-1, m}\right) \text {. }
\end{aligned}
$$


In Eqs. $(53,55)$, the expression in the first set of parenthesis are the $\beta_{s} w_{\ell, m}$ and $\alpha_{s} w_{\ell, m}$ of $\left({ }_{s} Y_{\ell, m} \rightarrow{ }_{s+1} Y_{\ell, m}\right)$, the expression in the second set of parenthesis are the $\beta_{s} w_{\ell+1, m}$ and $\alpha_{s} w_{\ell+1, m}$ of $\left({ }_{s} Y_{\ell+1, m} \rightarrow{ }_{s+1} Y_{\ell, m}\right)$, the expression in the third set of parenthesis are the $\beta_{s} w_{\ell-1, m}$ and $\alpha_{s} w_{\ell-1, m}$ of $\left({ }_{s} Y_{\ell-1, m} \rightarrow{ }_{s+1} Y_{\ell, m}\right)$, the expression in the fourth set of parenthesis are the $\beta_{s} w_{\ell, m}$ and $\alpha_{s} w_{\ell, m}$ of $\left({ }_{s} Y_{\ell, m} \rightarrow{ }_{s+1} Y_{\ell+1, m}\right)$, and the expression in the fifth set of parenthesis are the $\beta_{s} w_{\ell, m}$ and $\alpha_{s} w_{\ell, m}$ of $\left({ }_{s} Y_{\ell, m} \rightarrow{ }_{s+1} Y_{\ell-1, m}\right)$. To get the $\beta$ 's and $\alpha$ 's of the last four expressions, we will need the following relations,

$$
\begin{aligned}
& { }_{s+1} Y_{\ell, m}=\sqrt{\frac{2 \ell+1}{2 \ell+3}} \frac{-(\ell+s+1)[m+(\ell+1) \cos \theta]}{\sqrt{\left[(\ell+1)^{2}-m^{2}\right]\left[(\ell+1)^{2}-s^{2}\right](\ell-s)(\ell+s+1)}} \\
& \times\left[\partial_{\theta}-\frac{m(\ell+s+1) \cot \theta}{[m+(\ell+1) \cos \theta]}-\frac{(\ell+1)^{2}(s-1) \cos \theta \cot \theta}{(\ell+s+1)[m+(\ell+1) \cos \theta]}\right. \\
& -\frac{\left[\ell^{2}+s^{2}+m^{2}(s+1)+\ell\left(m^{2}+s^{2}+2\right)+1\right] \csc \theta}{(\ell+s+1)[m+(\ell+1) \cos \theta]} \\
& \left.+\frac{(\ell+1)^{2}(\ell+2) \sin \theta}{(\ell+s+1)[m+(\ell+1) \cos \theta]}\right]{ }_{s} Y_{\ell+1, m} \\
& { }_{s+1} Y_{\ell, m}=\sqrt{\frac{2 \ell+1}{2 \ell-1}} \frac{-(\ell-s)(-m+\ell \cos \theta)}{\sqrt{\left(\ell^{2}-m^{2}\right)\left(\ell^{2}-s^{2}\right)(\ell-s)(\ell+s+1)}}\left[\partial_{\theta}-\frac{m(\ell-s) \cot \theta}{(-m+\ell \cos \theta)}\right. \\
& \left.-\frac{\left(\ell^{2}-2 m^{2}+2 \ell s\right) \csc \theta}{2(-m+\ell \cos \theta)}+\frac{\ell^{2} \cos 2 \theta \csc \theta}{2(-m+\ell \cos \theta)}\right]_{s} Y_{\ell-1, m} \\
& { }_{s+1} Y_{\ell+1, m}=\sqrt{\frac{2 \ell+3}{2 \ell+1}} \frac{-\sqrt{\ell-s+1}[-m+(\ell+1) \cos \theta]}{\sqrt{\left[(\ell+1)^{2}-m^{2}\right]\left[(\ell+1)^{2}-s^{2}\right](\ell+s+2)}} \\
& {\left[\partial_{\theta}-\frac{m(\ell-s+1) \cot \theta}{[-m+(\ell+1) \cos \theta]}-\frac{(\ell+1)^{2}(s-1) \cos \theta \cot \theta}{(\ell-s+1)[-m+(\ell+1) \cos \theta]}\right.} \\
& +\frac{\left[s^{2}(\ell+1)-\left(\ell-m^{2}+1\right)(\ell+1)-m^{2} s\right] \csc \theta}{(\ell-s+1)[-m+(\ell+1) \cos \theta]} \\
& \left.-\frac{\ell(\ell+1)^{2} \sin \theta}{(\ell-s+1)[-m+(\ell+1) \cos \theta]}\right]{ }_{s} Y_{\ell, m} \\
& { }_{s+1} Y_{\ell-1, m}=\sqrt{\frac{2 \ell-1}{2 \ell+1}} \frac{-(\ell+s)(m+\ell \cos \theta)}{\sqrt{\left(\ell^{2}-m^{2}\right)\left(\ell^{2}-s^{2}\right)(\ell+s)(\ell-s-1)}}\left[\partial_{\theta}-\frac{m(\ell+s) \cot \theta}{(m+\ell \cos \theta)}\right. \\
& \left.+\frac{\left(\ell^{2}-2 m^{2}-2 \ell s\right) \csc \theta}{2(m+\ell \cos \theta)}-\frac{\ell^{2} \cos 2 \theta \csc \theta}{2(m+\ell \cos \theta)}\right]{ }_{s} Y_{\ell, m}
\end{aligned}
$$


The results are as follows:

$$
\begin{aligned}
& \beta_{(s, \ell, m) \rightarrow(s+1, \ell, m)}=\frac{-1}{\sqrt{(\ell-s)(\ell+s+1)}}, \\
& \beta_{(s, \ell+1, m) \rightarrow(s+1, \ell, m)}=\sqrt{\frac{2 \ell+1}{2 \ell+3}} \frac{-(\ell+s+1)[m+(\ell+1) \cos \theta]}{\sqrt{\left[(\ell+1)^{2}-m^{2}\right]\left[(\ell+1)^{2}-s^{2}\right](\ell-s)(\ell+s+1)}}, \\
& \beta_{(s, \ell-1, m) \rightarrow(s+1, \ell, m)}=\sqrt{\frac{2 \ell+1}{2 \ell-1}} \frac{-(\ell-s)(-m+\ell \cos \theta)}{\sqrt{\left(\ell^{2}-m^{2}\right)\left(\ell^{2}-s^{2}\right)(\ell-s)(\ell+s+1)}}, \\
& \beta_{(s, \ell, m) \rightarrow(s+1, \ell+1, m)}=\sqrt{\frac{2 \ell+3}{2 \ell+1}} \frac{-\sqrt{\ell-s+1}[-m+(\ell+1) \cos \theta]}{\sqrt{\left[(\ell+1)^{2}-m^{2}\right]\left[(\ell+1)^{2}-s^{2}\right](\ell+s+2)}}, \\
& \beta_{(s, \ell, m) \rightarrow(s+1, \ell-1, m)}=\sqrt{\frac{2 \ell-1}{2 \ell+1}} \frac{-(\ell+s)(m+\ell \cos \theta)}{\sqrt{\left(\ell^{2}-m^{2}\right)\left(\ell^{2}-s^{2}\right)(\ell+s)(\ell-s-1)}}, \\
& \alpha_{(s, \ell, m) \rightarrow(s+1, \ell, m)}=\frac{1}{\sqrt{(\ell-s)(\ell+s+1)}}\left[\frac{m}{\sin \theta}+\left(s+\frac{1}{2}\right) \cot \theta\right], \\
& \alpha_{(s, \ell+1, m) \rightarrow(s+1, \ell, m)}=\sqrt{\frac{2 \ell+1}{2 \ell+3}} \frac{-(\ell+s+1)[m+(\ell+1) \cos \theta]}{\sqrt{\left[(\ell+1)^{2}-m^{2}\right]\left[(\ell+1)^{2}-s^{2}\right](\ell-s)(\ell+s+1)}} \\
& \times\left[-\frac{m(\ell+s+1) \cot \theta}{[m+(\ell+1) \cos \theta]}-\frac{(\ell+1)^{2}(s-1) \cos \theta \cot \theta}{(\ell+s+1)[m+(\ell+1) \cos \theta]}\right. \\
& -\frac{\left[\ell^{2}+s^{2}+m^{2}(s+1)+\ell\left(m^{2}+s^{2}+2\right)+1\right] \csc \theta}{(\ell+s+1)[m+(\ell+1) \cos \theta]} \\
& \left.+\frac{(\ell+1)^{2}(\ell+2) \sin \theta}{(\ell+s+1)[m+(\ell+1) \cos \theta]}\right] \text {, } \\
& \alpha_{(s, \ell-1, m) \rightarrow(s+1, \ell, m)}=\sqrt{\frac{2 \ell+1}{2 \ell-1}} \frac{-(\ell-s)(-m+\ell \cos \theta)}{\sqrt{\left(\ell^{2}-m^{2}\right)\left(\ell^{2}-s^{2}\right)(\ell-s)(\ell+s+1)}} \\
& {\left[-\frac{m(\ell-s) \cot \theta}{(-m+\ell \cos \theta)}-\frac{\left(\ell^{2}-2 m^{2}+2 \ell s\right) \csc \theta}{2(-m+\ell \cos \theta)}+\frac{\ell^{2} \cos 2 \theta \csc \theta}{2(-m+\ell \cos \theta)}\right],} \\
& \alpha_{(s, \ell, m) \rightarrow(s+1, \ell+1, m)}=\sqrt{\frac{2 \ell+3}{2 \ell+1}} \frac{-\sqrt{\ell-s+1}[-m+(\ell+1) \cos \theta]}{\sqrt{\left[(\ell+1)^{2}-m^{2}\right]\left[(\ell+1)^{2}-s^{2}\right](\ell+s+2)}} \\
& {\left[-\frac{m(\ell-s+1) \cot \theta}{[-m+(\ell+1) \cos \theta]}-\frac{(\ell+1)^{2}(s-1) \cos \theta \cot \theta}{(\ell-s+1)[-m+(\ell+1) \cos \theta]}\right.} \\
& +\frac{\left[s^{2}(\ell+1)-\left(\ell-m^{2}+1\right)(\ell+1)-m^{2} s\right] \csc \theta}{(\ell-s+1)[-m+(\ell+1) \cos \theta]} \\
& \left.-\frac{\ell(\ell+1)^{2} \sin \theta}{(\ell-s+1)[-m+(\ell+1) \cos \theta]}\right] \text {, }
\end{aligned}
$$




$$
\begin{aligned}
\alpha_{(s, \ell, m) \rightarrow(s+1, \ell-1, m)=} & \sqrt{\frac{2 \ell-1}{2 \ell+1}} \frac{-(\ell+s)(m+\ell \cos \theta)}{\sqrt{\left(\ell^{2}-m^{2}\right)\left(\ell^{2}-s^{2}\right)(\ell+s)(\ell-s-1)}} \\
& {\left[-\frac{m(\ell+s) \cot \theta}{(m+\ell \cos \theta)}+\frac{\left(\ell^{2}-2 m^{2}-2 \ell s\right) \csc \theta}{2(m+\ell \cos \theta)}-\frac{\ell^{2} \cos 2 \theta \csc \theta}{2(m+\ell \cos \theta)}\right] . }
\end{aligned}
$$

Using the above relations, we obtain:

$$
{ }_{s+1} S_{\ell, m}^{\gamma}=\left({ }_{s} \beta_{\ell, m}^{\gamma} \partial_{\theta}+{ }_{s} \alpha_{\ell, m}^{\gamma}\right)_{s} S_{\ell, m}^{\gamma}+O\left(\gamma^{2}\right)
$$

where

$$
\begin{aligned}
{ }_{s} \beta_{\ell, m}^{\gamma}= & \frac{-1}{\sqrt{(\ell-s)(\ell+s+1)}}-\frac{m\left(\ell^{2}+\ell+2 s+1\right) \gamma}{\ell^{2}(\ell+1)^{2} \sqrt{(\ell-s)(\ell+s+1)}} \\
& -\frac{(2 s+1) \cos \theta \gamma}{\ell(\ell+1) \sqrt{(\ell-s)(\ell+s+1)}} \\
{ }_{s} \alpha_{\ell, m}^{\gamma}= & \frac{m \csc \theta+s \cot \theta}{\sqrt{(\ell-s)(\ell+s+1)}}+\frac{m\left[\ell(\ell+1)+3 \ell s(\ell+1)+2 s^{2}+s\right] \cot \theta \gamma}{\ell^{2}(\ell+1)^{2} \sqrt{(\ell-s)(\ell+s+1)}} \\
& +\frac{\left[\ell s(\ell+1)(2 s+1)+m^{2}\left(\ell^{2}+\ell+2 s+1\right)\right] \csc \theta \gamma}{\ell^{2}(\ell+1)^{2} \sqrt{(\ell-s)(\ell+s+1)}} \\
& -\frac{\sin \theta \gamma}{\sqrt{(\ell-s)(\ell+s+1)}}
\end{aligned}
$$

Finally, using the relation,

$$
{ }_{-s} S_{\ell,-m}^{-\gamma}=(-1)^{m+s}{ }_{s} S_{\ell, m}^{\gamma}
$$

we identify the lowering operator:

$$
{ }_{s-1} S_{\ell, m}^{\gamma}=-\left({ }_{-s} \beta_{\ell,-m}^{-\gamma} \partial_{\theta}+{ }_{-s} \alpha_{\ell,-m}^{-\gamma}\right)_{s} S_{\ell, m}^{\gamma}+O\left(\gamma^{2}\right) .
$$

\section{Conclusion}

Equations (61) and (65) represent the final result of our work-equations for the linearin- $\gamma$ spin-raising and lowering operators for spin-weighted spheroidal harmonics. We have obtained these, and Eqs. (62) and (63) for the required ${ }_{s} \alpha_{\ell, m}^{\gamma}$ and ${ }_{s} \beta_{\ell, m}^{\gamma}$, using methods described in Sect. 2 and which can be readily extended to higher order in $\gamma$, if required. Some special cases, corresponding to $s= \pm \ell$ and/or $m= \pm \ell$, are dealt with further in the Appendix.

Acknowledgments This work was supported in part by the European Research Council under the European Union's Seventh Framework Programme (FP7/2007-2013)/ERC Grant agreement No. 304978 to the University of Southampton, and NSF Grants PHY 1205906 and PHY 1314529 to the University of 
Florida. Hospitality for BFW at the University of Southampton at an early stage in this work is gratefully acknowledged. Support from the CNRS through the IAP, where part of this work was carried out, is also acknowledged, as well as support from the French state funds managed by the ANR within the Investissements d'Avenir programme under Grant No. ANR-11-IDEX-0004-02.

Open Access This article is distributed under the terms of the Creative Commons Attribution 4.0 International License (http://creativecommons.org/licenses/by/4.0/), which permits unrestricted use, distribution, and reproduction in any medium, provided you give appropriate credit to the original author(s) and the source, provide a link to the Creative Commons license, and indicate if changes were made.

\section{Appendix : Building ${ }_{s} T_{\ell, m}^{\gamma}$ for a special cases}

For cases such as $m=\ell, s=-\ell$ or $m=\ell=s$, one cannot simply use the general form,

$$
{ }_{s} T_{\ell, m}^{\gamma}={ }_{s} X_{\ell, m}+\gamma_{s} b_{\ell, \ell+1}^{m} X_{\ell+1, m}+\gamma_{s} b_{\ell, \ell-1}^{m} X_{\ell-1, m},
$$

with ${ }_{s} b_{\ell, \ell-1}^{m}=0$ as when building ${ }_{s} S_{\ell, m}^{\gamma}$ for these cases. Instead, it then becomes imperative to carefully look at the analyticity of the Clebsch-Gordan coefficients in ${ }_{s} b_{\ell, \ell-1}^{m}$, the coefficients of $\partial^{s} Q_{\ell-1}^{m}$, and at times even multiplying them with extra coefficients so that ${ }_{s} T_{\ell, m}^{\gamma}$ for such cases satisfy Eq. (1).

In general cases, it is straight forward to use

$$
\begin{gathered}
{ }_{s} b_{\ell, \ell^{\prime}}^{m}=2 s\left(\frac{2 \ell+1}{2 \ell^{\prime}+1}\right)^{1 / 2} \frac{\left\langle\ell, m ; 1,0 \mid \ell^{\prime}, m\right\rangle\left\langle\ell,-s ; 1,0 \mid \ell^{\prime},-s\right\rangle}{\ell(\ell+1)-\ell^{\prime}\left(\ell^{\prime}+1\right)}, \text { and } \\
{ }_{s} X_{\ell, m}(\theta)=\sqrt{\frac{(2 \ell+1)(\ell-m) !}{4 \pi(\ell+m) !}} \partial^{s} Q_{\ell}^{m}(\cos \theta)
\end{gathered}
$$

where $\langle\mid\rangle$ are the Clebsch-Gordan coefficients which can be calculated using

$$
\begin{aligned}
& \left\langle j_{1}, m_{1} ; j_{2}, m_{2} \mid j, m\right\rangle=\sqrt{\frac{\left(j_{1}+j_{2}-j\right) !\left(j+j_{1}-j_{2}\right) !\left(j+j_{2}-j_{1}\right) !(2 j+1)}{\left(j+j_{1}+j_{2}+1\right) !}} \\
& \times \sum_{k=-\infty}^{\infty} \frac{(-1)^{k} \sqrt{\left(j_{1}+m_{1}\right) !\left(j_{1}-m_{1}\right) !\left(j_{2}+m_{2}\right) !\left(j_{2}-m_{2}\right) !(j+m) !(j-m) !}}{k !\left(j_{1}+j_{2}-j-k\right) !\left(j_{1}-m_{1}-k\right) !\left(j_{2}+m_{2}-k\right) !\left(j-j_{2}+m_{1}+k\right) !\left(j-j_{1}-m_{2}+k\right) !}
\end{aligned}
$$

Now, we study the following special cases. 


\section{$m=\ell$ and $|s| \neq \ell$}

In this case, there is a cancellation in the last term of the right-hand-side of Eq. (66). The ${ }_{s} b_{\ell, \ell-1}^{m}$ includes $\langle\ell, m ; 1,0 \mid \ell-1, m\rangle$ which go to zero as

$$
\frac{(2 m-1)}{\sqrt{2(2 m+1)}} \sqrt{(\ell-m)}+O(\ell-m)^{3 / 2}
$$

And $\sqrt{(\ell-1-m) !}$ in the numerator of the square-root of Eq. (68) blows up as $\frac{1}{\sqrt{(\ell-m)}}$ which cancels the one above. Using these and after empirically finding the coefficient $c_{\ell}$, we have

$$
{ }_{s} T_{\ell, m}^{\gamma}={ }_{s} X_{\ell, m}+\gamma{ }_{s} b_{\ell, \ell+1}^{m} X_{\ell+1, m}+\gamma c_{\ell}{ }_{s} \tilde{b}_{\ell, \ell-1 s}^{m} \tilde{X}_{\ell-1, m}+O\left(\gamma^{2}\right)
$$

where

$$
\begin{gathered}
c_{\ell}=\frac{-2}{2 \ell-1}, \\
{ }_{s} \tilde{b}_{\ell, \ell-1}^{m}=2 s\left(\frac{2 \ell+1}{2 \ell-1}\right)^{1 / 2} \frac{\frac{2 m-1}{\sqrt{2(2 m+1)}}\langle\ell,-s ; 1,0 \mid \ell-1,-s\rangle}{\ell(\ell+1)-\ell(\ell-1)}, \text { and } \\
\tilde{X}_{\ell-1, m}=\sqrt{\frac{2 \ell-1}{4 \pi(\ell-1+m) !}} \partial^{s} Q_{\ell}^{m} .
\end{gathered}
$$

\section{$m=-\ell$ and $|s| \neq \ell$}

The ${ }_{s} b_{\ell, \ell-1}^{m}$ includes $\langle\ell, m ; 1,0 \mid \ell-1, m\rangle$ which goes to zero as

$$
-\sqrt{\frac{2}{1-2 m}} \sqrt{(\ell+m)}+O(\ell+m)^{3 / 2} .
$$

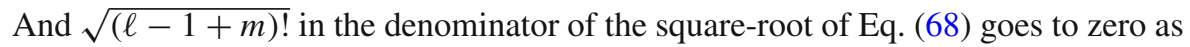
$\sqrt{(\ell+m)} .{ }_{s} X_{\ell, m}$ for $m=-\mu$ and $\ell=\mu-1$ (where $\mu>0$ ) blows up to leading order as

$$
(-1)^{s} \frac{\sqrt{(\mu+s-1) !(\mu-s-1) !} \tan ^{s}(\theta / 2) Q_{\mu-1}^{\mu}(\cos \theta)}{(\mu-1) !((2 \mu-1) !)^{3 / 2}(\ell+m)^{3 / 2}} .
$$

The coefficient $c_{\ell}$ is found empirically to be $(-1)^{\ell} \sqrt{(2 \ell-1) !} \sqrt{(\ell+m)}$. Using these we get

$$
{ }_{s} T_{\ell, m}^{\gamma}={ }_{s} X_{\ell, m}+\gamma{ }_{s} b_{\ell, \ell+1}^{m} X_{\ell+1, m}+\gamma c_{\ell s} \tilde{b}_{\ell, \ell-1}^{m} \tilde{X}_{\ell-1, m}+O\left(\gamma^{2}\right)
$$


where

$$
\begin{aligned}
c_{\ell} & =(-1)^{\ell} \sqrt{(2 \ell-1) !}, \\
{ }_{s} \tilde{b}_{\ell, \ell-1}^{m} & =2 s\left(\frac{2 \ell+1}{2 \ell-1}\right)^{1 / 2} \frac{-\sqrt{\frac{2}{1-2 m}}}{\ell(\ell+1)-\ell(\ell-1)}-s ; 1,0|\ell-1,-s\rangle \\
{ }_{s} \tilde{X}_{\ell-1, m} & =(-1)^{s} \sqrt{\frac{(2 \ell-1)(\ell-m-1) !}{4 \pi}} \\
& \frac{\sqrt{(s-m-1) !(-m-s-1) !} \tan ^{s}(\theta / 2) Q_{-m-1}^{-m}(\cos \theta)}{(-m-1) !((-2 m-1) !)^{3 / 2}}
\end{aligned}
$$

where appropriate powers of $\sqrt{\ell+m}$ in each of $c_{\ell},{ }_{s} \tilde{b}_{\ell, \ell-1}^{m}$ and ${ }_{s} \tilde{X}_{\ell-1, m}$ have been cancelled out to give a finite contribution.

\section{$|m| \neq \ell$ and $s=\ell$}

The ${ }_{s} b_{\ell, \ell-1}^{m}$ includes $\langle\ell,-s ; 1,0 \mid \ell-1,-s\rangle$ which, in the limit $\ell \rightarrow s$, goes to zero as

$$
-\sqrt{\frac{2}{1+2 s}} \sqrt{(\ell-s)}+O(\ell-s)^{3 / 2}
$$

and $ð^{s} Q_{\ell-1}^{m}(\theta)$ blows up as

$$
\frac{(-1)^{m}}{2^{s}} \sqrt{\frac{(2 s-2) ! !}{(2 s-1) ! !}} \frac{(s+m-1) !}{(s-1) !} \frac{\csc ^{s+m}\left(\frac{\theta}{2}\right) \sec ^{s-m}\left(\frac{\theta}{2}\right)}{\sqrt{\ell-s}} .
$$

Factors of $\sqrt{\ell-s}$ in the above two equations cancel each other and ${ }_{s} T_{\ell, m}^{\gamma}$ is then given by

$$
{ }_{s} T_{\ell, m}^{\gamma}={ }_{s} X_{\ell, m}+\gamma{ }_{s} b_{\ell, \ell+1}^{m} X_{\ell+1, m}+\gamma{ }_{s} \tilde{b}_{\ell, \ell-1}^{m}{ }_{X} \tilde{X}_{\ell-1, m}+O\left(\gamma^{2}\right)
$$

where

$$
\begin{aligned}
{ }_{s} \tilde{b}_{\ell, \ell-1}^{m}= & 2 s\left(\frac{2 \ell+1}{2 \ell-1}\right)^{1 / 2} \frac{-\sqrt{\frac{2}{1+2 s}}}{\ell(\ell+1)-\ell(\ell-1)}, m ; 1,0|\ell-1, m\rangle \\
{ }_{s} \tilde{X}_{\ell-1, m}= & \sqrt{\frac{(2 \ell-1)(\ell-1-m) !}{4 \pi(\ell-1+m) !} \frac{(-1)^{m}}{2^{s}}} \sqrt{\frac{(2 s-2) ! !}{(2 s-1) ! !}} \frac{(s+m-1) !}{(s-1) !} \\
& \csc ^{s+m}\left(\frac{\theta}{2}\right) \sec ^{s-m}\left(\frac{\theta}{2}\right) .
\end{aligned}
$$




\section{$|m| \neq \ell$ and $s=-\ell$}

The ${ }_{s} b_{\ell, \ell-1}^{m}$ includes $\langle\ell,-s ; 1,0 \mid \ell-1,-s\rangle$ which, in the limit $\ell \rightarrow-s$, goes to zero as

$$
\frac{-(2 s+1)}{\sqrt{2} \sqrt{1-2 s}} \sqrt{(\ell+s)}+O(\ell+s)^{3 / 2}
$$

and $ð^{s} Q_{\ell-1}^{m}(\theta)$ blows up as

$$
\frac{(-1)^{s}}{2^{|s|}} \sqrt{\frac{(2|s|-2) ! !}{(2|s|-1) ! !} \frac{(|s|+m-1) ! \csc ^{|s|-m}\left(\frac{\theta}{2}\right) \sec ^{|s|+m}\left(\frac{\theta}{2}\right)}{\sqrt{\ell+s}}}
$$

The $\sqrt{\ell+s}$ in the above two equations cancel each other and ${ }_{s} T_{\ell, m}^{\gamma}$ is then given by

$$
{ }_{s} T_{\ell, m}^{\gamma}={ }_{s} X_{\ell, m}+\gamma{ }_{s} b_{\ell, \ell+1}^{m} X_{\ell+1, m}+\gamma c_{\ell s} \tilde{b}_{\ell, \ell-1}^{m} \tilde{X}_{\ell-1, m}+O\left(\gamma^{2}\right)
$$

where

$$
\begin{gathered}
c_{\ell}=\frac{-2}{2 \ell-1} \\
\tilde{b}_{\ell, \ell-1}^{m}=2 s\left(\frac{2 \ell+1}{2 \ell-1}\right)^{1 / 2} \frac{\frac{-(2 s+1)}{\sqrt{2} \sqrt{1-2 s}}}{\ell(\ell+1)-\ell(\ell-1)}\{, 1,0|\ell-1, m\rangle \\
{ }_{s} \tilde{X}_{\ell-1, m}=\sqrt{\frac{(2 \ell-1)(\ell-1-m) !}{4 \pi(\ell-1+m) !} \frac{(-1)^{s}}{2^{|s|}} \sqrt{\frac{(2|s|-2) ! !}{(2|s|-1) ! !} \frac{(|s|+m-1) !}{(|s|-1) !}}} \\
\csc ^{|s|-m}\left(\frac{\theta}{2}\right) \sec ^{|s|+m}\left(\frac{\theta}{2}\right) .
\end{gathered}
$$

$m=\ell$ and $s=\ell$

This case is a hybrid form of the $s=\ell$ and the $m=\ell$ cases. The two Clebsch-Gordan coefficients in ${ }_{s} b_{\ell, \ell-1}^{m}$, and the denominator in the square-root of the coefficient of $\partial^{s} Q_{\ell-1}^{m}$ have the same form as the $s=\ell$ and $m=\ell$ cases. The coefficient $c_{\ell}$ is also same as the one in the $m=\ell$ case. $\check{\partial}^{s} Q_{\ell-1}^{m}$ blows up as

$$
(-1) \frac{\sqrt{(2 s-1) !}}{2 \sqrt{\ell-s}} \csc ^{2 s}(\theta / 2)
$$

which cancels the Clebsch-Gordan coefficient containing $s$. We then have

$$
{ }_{s} T_{\ell, m}^{\gamma}={ }_{s} X_{\ell, m}+\gamma{ }_{s} b_{\ell, \ell+1}^{m} X_{\ell+1, m}+\gamma c_{\ell s} \tilde{b}_{\ell, \ell-1 s}^{m} \tilde{X}_{\ell-1, m}
$$


where

$$
\begin{gathered}
c_{\ell}=\frac{-2}{2 \ell-1}, \\
{ }_{s} \tilde{b}_{\ell, \ell-1}^{m}=2 s\left(\frac{2 \ell+1}{2 \ell-1}\right)^{1 / 2} \frac{\frac{2 m-1}{\sqrt{2(2 m+1)}} \frac{-\sqrt{2}}{\sqrt{2 s+1}}}{\ell(\ell+1)-\ell(\ell-1)}, \text { and } \\
{ }_{s} \tilde{X}_{\ell-1, m}=\sqrt{\frac{2 \ell-1}{4 \pi(\ell-1+m) !}}(-1)^{s} \frac{\sqrt{(2 s-1) !}}{2} \csc ^{2 s}(\theta / 2) .
\end{gathered}
$$

\section{$m=\ell$ and $s=-\ell$}

This case is a hybrid form of the $s=-\ell$ and the $m=\ell$ cases. The two Clebsch-Gordan coefficients in ${ }_{s} b_{\ell, \ell-1}^{m}$, and the denominator in the square-root of the coefficient of $\mathrm{\partial}^{s} Q_{\ell-1}^{m}$ have the same form as the $s=-\ell$ and $m=\ell$ cases. $\partial^{s} Q_{\ell-1}^{m}$ blows up as

$$
(-1)^{s} \frac{\sqrt{(-2 s-1) !}}{2 \sqrt{\ell+s}} \sec ^{-2 s}(\theta / 2)
$$

which cancels the Clebsch-Gordan coefficient containing $s$. We then have

$$
{ }_{s} T_{\ell, m}^{\gamma}={ }_{s} X_{\ell, m}+\gamma{ }_{s} b_{\ell, \ell+1 s}^{m} X_{\ell+1, m}+\gamma c_{\ell s} \tilde{b}_{\ell, \ell-1 s}^{m} \tilde{X}_{\ell-1, m}
$$

where

$$
\begin{gathered}
c_{\ell}=\left(\frac{-2}{2 \ell-1}\right)^{2}, \\
{ }_{s} \tilde{b}_{\ell, \ell-1}^{m}=2 s\left(\frac{2 \ell+1}{2 \ell-1}\right)^{1 / 2} \frac{\frac{2 m-1}{\sqrt{2(2 m+1)}} \frac{-(2 s+1)}{\sqrt{2} \sqrt{1-2 s}}}{\ell(\ell+1)-\ell(\ell-1)}, \text { and } \\
{ }_{s} \tilde{X}_{\ell-1, m}=\sqrt{\frac{2 \ell-1}{4 \pi(\ell-1+m) !}}(-1)^{s} \frac{\sqrt{(-2 s-1) !}}{2} \cos ^{2 s}(\theta / 2) .
\end{gathered}
$$

\section{$m=-\ell$ and $s=\ell$}

This case is a hybrid of the $m=-\ell$ and the $s=\ell$ cases. We use $\epsilon$ to denote the small factor $(\ell+m)$ [or $(\ell-s)$ ]. The two Clebsch-Gordan coefficients in ${ }_{s} b_{\ell, \ell-1}^{m}$ go to zero as $\epsilon^{1 / 2}$ each, and the $\sqrt{(\ell-1+m)}$ in the expression of ${ }_{s} X_{\ell-1, m}$ goes to zero as $\epsilon^{1 / 2}$. $\partial^{s} Q_{\ell-1}^{m}$ blows up as $\epsilon^{-2}$ and we empirically find that the coefficient ${ }_{s} c_{\ell}$ is $(-1)^{s} \sqrt{(2 \ell-1) !} \epsilon^{1 / 2}$ canceling the extra $\epsilon^{-1 / 2}$ left in $\partial^{s} Q_{\ell-1}^{m}$. Here $\epsilon$ is the small factor $(\ell+m)$ [or $(\ell-s)]$. We then have

$$
{ }_{s} T_{\ell, m}^{\gamma}={ }_{s} X_{\ell, m}+\gamma{ }_{s} b_{\ell, \ell+1}^{m} X_{\ell+1, m}+\gamma_{s} c_{\ell s} \tilde{b}_{\ell, \ell-1}^{m} \tilde{X}_{\ell-1, m}
$$


where

$$
\begin{gathered}
{ }_{s} c_{\ell}=(-1)^{s} \sqrt{(2 \ell-1) !}, \\
{ }_{s} \tilde{b}_{\ell, \ell-1}^{m}=2 s\left(\frac{2 \ell+1}{2 \ell-1}\right)^{1 / 2} \frac{\frac{-\sqrt{2}}{\sqrt{1-2 m}} \frac{-\sqrt{2}}{\sqrt{2 s+1}}}{\ell(\ell+1)-\ell(\ell-1)}, \text { and } \\
{ }_{s} \tilde{X}_{\ell-1, m}=\sqrt{\frac{(2 \ell-1)(\ell-1-m) !}{4 \pi}} \frac{1}{2(2 s-1) !} \sec ^{2 s}(\theta / 2) .
\end{gathered}
$$

$m=-\ell$ and $s=-\ell$

Identical cancellations take place as in the previous case. We have

$$
{ }_{s} T_{\ell, m}^{\gamma}={ }_{s} X_{\ell, m}+\gamma{ }_{s} b_{\ell, \ell+1}^{m} X_{\ell+1, m}+\gamma_{s} c_{\ell s} \tilde{b}_{\ell, \ell-1}^{m} \tilde{X}_{\ell-1, m}
$$

where

$$
\begin{gathered}
{ }_{s} c_{\ell}=(-1)^{s+1} \frac{2 \sqrt{(2 \ell-1) !}}{(2 \ell-1)}, \\
{ }_{s} \tilde{b}_{\ell, \ell-1}^{m}=2 s\left(\frac{2 \ell+1}{2 \ell-1}\right)^{1 / 2} \frac{\frac{-\sqrt{2}}{\sqrt{1-2 m}} \frac{-(1+2 s)}{\sqrt{2} \sqrt{1-2 s}}}{\ell(\ell+1)-\ell(\ell-1)} \\
{ }_{s} \tilde{X}_{\ell-1, m}=\sqrt{\frac{(2 \ell-1)(\ell-1-m) !}{4 \pi}} \frac{1}{2(2|s|-1) !} \csc ^{2|s|}(\theta / 2) .
\end{gathered}
$$

\section{References}

1. Goldberg, J.N., MacFarlane, A.J., Newman, E.T., Rohrlich, F., Sudarshan, E.C.G.: Spin-s spherical harmonics and ð. J. Math. Phys. 8, 2155 (1967)

2. Teukolsky, S.: Rotating black holes-separable wave equations for gravitational and electromagnetic perturbations. Phys. Rev. Lett. 29, 1114-1118 (1972)

3. Teukolsky, S.A.: Perturbations of a rotating black hole. 1. Fundamental equations for gravitational electromagnetic and neutrino field perturbations. Astrophys. J. 185, 635-647 (1973)

4. Flammer, C.: Spheroidal Wave Functions. Stanford Univ. Press, Stanford, CA (1957)

5. Leaver, E.W.: Solutions to a generalized spheroidal wave equation: Teukolsky's equations in general relativity, and the two-center problem in molecular quantum mechanics. J. Math. Phys. 27(5), 12381265 (1986)

6. Casals, M., Ottewill, A.C.: Canonical quantization of the electromagnetic field on the Kerr background. Phys. Rev. D 71, 124,016 (2005)

7. Casals, M., Kanti, P., Winstanley, E.: Brane decay of a (4+n)-dimensional rotating black hole ii: spin-1 particles. J. High Energy Phys. 2006(02), 051 (2006)

8. Berti, E., Cardoso, V., Casals, M.: Eigenvalues and eigenfunctions of spin-weighted spheroidal harmonics in four and higher dimensions. Phys. Rev. D 73, 024,013 (2006)

9. Press, W.H., Teukolsky, S.A.: Perturbations of a rotating black hole. II. Dynamical stability of the Kerr metric. Astrophys. J. 185, 649-674 (1973)

10. Fackerell, E.D., Crossman, R.G.: Spin-weighted angular spheroidal functions. J. Math. Phys. 18(9), 1849-1854 (1977)

11. Whiting, B.F.: The relation of solutions of different ODEs is a commutation relation. N.-Holl. Math. Stud. 92, 561-570 (1984)

12. Whiting, B.F.: Mode stability of the Kerr black hole. J. Math. Phys. 30, 1301-1305 (1989) 